Ophthalmologica 1948;115:I-IV

\title{
Contents, Vol. 115, 1948
}

\section{Index}

Andrade, L. d', Importance de la zonulotomie directe pour

exécution d'une phacectomie totale 78

Auw-Yang Sien, A Case of Choroidal Apoplexy Diagnosed

as a Sarcoma of the Choroid 1

Bínkhorst, C. D., Toxoplasmosis (With Plate II and III) . 65 Biro, I., Data Concerning the Heredity of Astigmatism . 156 Busacca, A., et E. Pinticart de W., Etude gonioscopique d'un cas de Embryotoxon corneae posterius . . . 283 Carreras Matas, B., Aiigenhintergrunduntersuchung und Spaltlampenmikroskopie des Augenhintergrundes mittels Kontakt- und Vorlegeglas 179

Csillag, F., Bemerkung zur Arbeit von Jean Sedan und Simone Sédan-Bauby aus Ophthalmologica Vol. 114, S. 65186

Dekking, H. M., Opacity Meter for Cornea and Lens .. 219 Fanta, H., Die Wirkungsweise des Pilokarpin bei normalen

Augen 338

Ferrié, J.; A Propos des complications sensorielles et ner-

veuses de la fièvre récurrente cosmopolite . . . 227 Fieandt, O. von, Experiences on

Transplantation of Human

Vitreous 257

Gardilcic, A., Über eine neuartige Operation der Iridodialyse mittels Iris-Naht 141

- Ein Vorschlag einer Ptosisoperation durch Levatorfaltung 269

Gát, L., Lip Evertor for Mucous Membrane Grafting . . 47 Goedbloed, J., Capillary Hemorrhages of the Retina and

Capillary Fragility 174

Guggenbühl, A., Das stereoskopische Sehen des hell- und

dunkeladapt $1 / 8$ rten Auges 193

Hodel-Boos, Michaela, Untersuchungen über das Bewe-

gungssehen bei Hell- und Dunkeladaptation ... 25 Hruby, K., Weitere Vereínfachungen der Spaltlampenmikroskopie des hinteren Augenabschnittes .... 290 Jonkers, G. H., Seasonal Variations in the Occurrence of

Retinal Detachments 308, 316

Kurz, O., Ungewöhnliche Abflußhindernisse in den oberen

Tränenwegen 101

Miller, B., Kontrolle der therapeutischen Wirkung von

Priscol an den Netzhautgefäßen 
Much, V., Die Bienengift-Therapie bei Herpes corneae, Ke-

ratitis superficialis punctata simplex und epidemica . 89

Németh, L., Augenveränderungen bei tuberkulösen Haut-krankheiten, mit besonderer

Berücksichtigung des Au-genhintergrundes 167

Pinticart de W., E., vide Busucca, A.

Rosen, E., Some New Concepts concerning Ocular Complications following Vaccination 321

Roussel, F., vide Weekers, R.

Vos, T. A., Une resection simplifiée du droit externe ou

interne 22

- Iris numerata 354

Vrabec, Fr., Quatre cas de mélanoblastome de la choroïde

humaine cultivés in vitro 129

Weekers, R., et $\mathrm{F}$. Roussel, Les modifications de la frequence critique de fusion après guérison chirurgicaledu décollement rétinien 297

Weidmann, W., Ein einfaches, se $\Gamma$ bstregistrierendes Perimeter für genaue Untersuchungen 332

Winkelman, J. E., Angioid Streaks 84

GESELLSCHAFTSBERICHTE - SOCIETY TRANSACTIONS - SOCIÉTÉS

Report of the 111th Meeting of the Netherlands Ophthalmological

Society in Amsterdam, the 14/15 December 1946 49, 110

Jahresve sammlung der Fachgruppe der ungarischen Augenärzte. Budapest, 18./19. Oktober

1947 187

Vereinigung der Basler Augenärzte. Zusammenkunft am 12. Mai $1947 \quad 241$

Report of the 112th Meeting of the Netherlands Ophthalmological Society in the

Ophthalmological Department of the University Hospital («Academisch Ziekenhuis») in Leyden 358

B. BUCHBESPRECHUNGEN -BOOKS RE VIEW - LIVRES NOUVEAUX

64

192

255

319

380 